\title{
Pulmonary Capillaritis in Wegener's Granulomatosis Detected Via Transbronchial Lung Biopsy
}

\author{
Takashi Ishiguro ${ }^{1}$, Noboru Takayanagi ${ }^{1}$, Shozaburo Yamaguchi ${ }^{1}$, Yoshihiko Shimizu ${ }^{2}$, \\ Tsutomu Yanagisawa ${ }^{1}$, Yutaka Sugita ${ }^{1}$ and Yoshinori Kawabata ${ }^{2}$
}

\begin{abstract}
A 73-year-old woman presented to our hospital with scleritis, cough, and hemoptysis. Chest computed tomography showed a mass lesion, multiple ground-glass opacities, and nodules. Bronchoscopy showed a skip lesion of mucosal erosion with white necrotic substance; however, a biopsy specimen of the erosion yielded no characteristic findings. Transbronchial lung biopsy showed alveolar hemorrhage and capillaritis. Proteinase-3 antineutrophil cytoplasmic antibody was elevated to $62.5 \mathrm{U} / \mathrm{mL}$, and she was diagnosed as having Wegener's granulomatosis. Steroid therapy combined with cyclophosphamide was started; however, hemoptysis was not resolved until plasmapheresis therapy was added. Her general condition initially improved, but respiratory insufficiency worsened with fever on the 30th hospital day. The patient subsequently died on the 41 st hospital day, and cytomegalovirus infection was suggested as a possible cause of death.
\end{abstract}

Key words: Wegener's granulomatosis, transbronchial lung biopsy, capillaritis, diagnosis, diffuse alveolar hemorrhage

(Intern Med 51: 905-909, 2012)

(DOI: 10.2169/internalmedicine.51.6518)

\section{Introduction}

Wegener's granulomatosis (WG) is a systemic disease characterized by necrotizing vasculitis and granulomatous inflammation. It typically involves the upper airways, lungs, and kidneys, although the inflammatory destructive lesions may develop in any organ. WG causes diffuse alveolar hemorrhage (DAH) in $8 \%$ of patients, and DAH usually occurs as a consequence of capillaritis (1). The presence of DAH increases the early mortality of WG, and early diagnosis and treatment are essential (2). Because specimens obtained via transbronchial lung biopsy (TBLB) are small, detection of capillaritis via TBLB has been thought to be difficult. To our knowledge, there have been no reports in which capillaritis in WG was detected via TBLB. Here, we present a patient with WG in whom TBLB was useful in diagnosing DAH due to capillaritis.

\section{Case Report}

A 73-year-old woman developed nasal obstruction in November and scleritis in December 2005. Difficulty in hearing and dry cough developed in January and April 2006, respectively, and hemoptysis in May 2006, at which time she presented to our hospital and was admitted for further evaluation. She had never smoked, and she had no evident past history.

On admission, she had fever of $38.2{ }^{\circ} \mathrm{C}$. Physical examination showed no evident findings except for right-sided scleritis and hearing loss. Arterial partial pressure of oxygen was 70.5 Torr under ambient air. White blood cell count was increased to $16,600 / \mathrm{mm}^{3}$ (neutrophils $15,000 / \mathrm{mm}^{3}$, lymphocytes $700 / \mathrm{mm}^{3}$ ). Hemoglobin was decreased to $9.8 \mathrm{~g} / \mathrm{dL}$, and platelet count was $30.9 \times 10^{4} / \mathrm{mm}^{3}$. Blood urea nitrogen was elevated to $26 \mathrm{mg} / \mathrm{dL}$, serum creatinine was $0.7 \mathrm{mg} / \mathrm{dL}$, and C-reactive protein was elevated to $22.2 \mathrm{mg} / \mathrm{dL}$. Antinuclear antibody was slightly elevated 160 titers, and proteinase- 3

${ }^{1}$ Department of Respiratory Medicine, Saitama Cardiovascular and Respiratory Center, Japan and ${ }^{2}$ Department of Diagnostic Pathology, Saitama Cardiovascular and Respiratory Center, Japan

Received for publication September 12, 2011; Accepted for publication December 12, 2011

Correspondence to Dr. Takashi Ishiguro, ishiguro.takashi@pref.saitama.lg.jp 

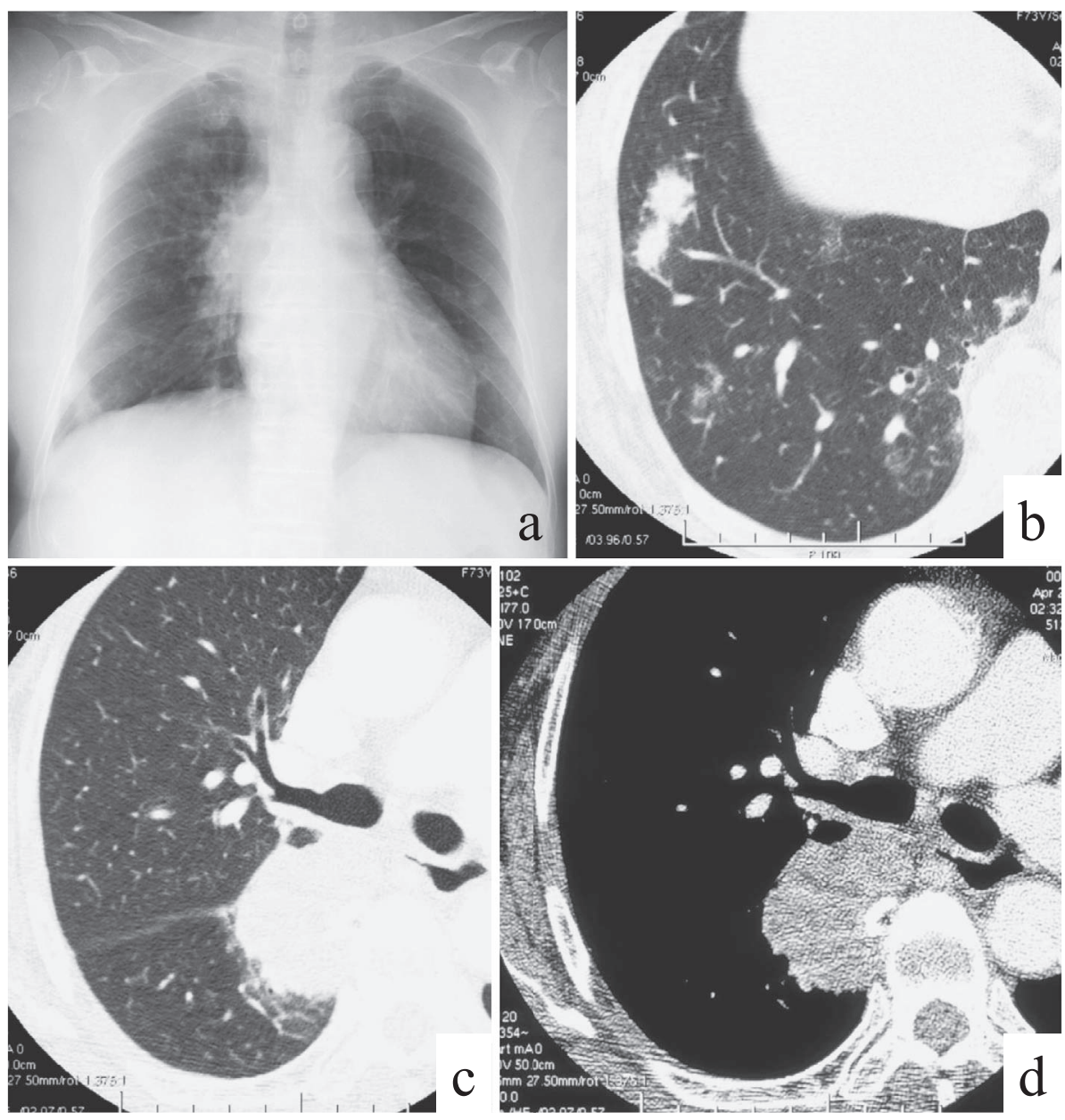

Figure 1. Radiological findings. Chest X-ray showed a nodular shadow in the right lower lung field and right-sided protrusion of the hilum (a). Chest computed tomography showed multiple ground-glass opacities and nodules were found in both lung fields (b). Chest computed tomography showed a mass lesion of $55 \mathrm{~mm}$ in diameter in the superior segment of the right lower lobe (c, d).
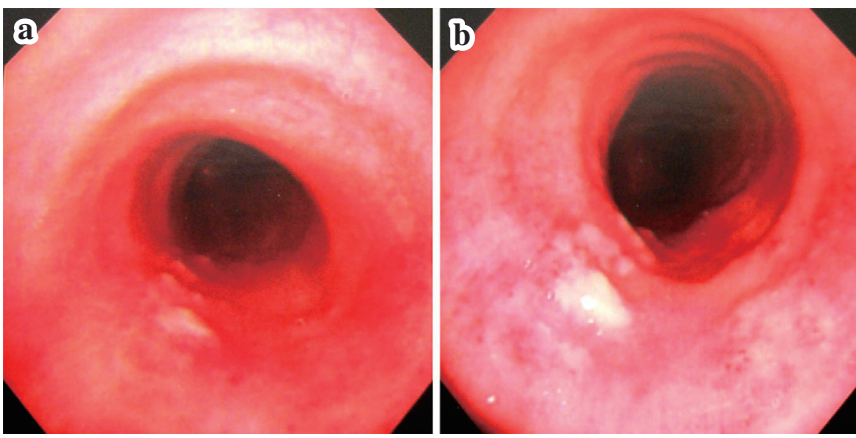

Figure 2. Bronchoscopic findings. A skip lesion of mucosal erosion with white necrotic substance was seen (a, b).

antineutrophil cytoplasmic antibody (PR-3 ANCA) was elevated to $62.5 \mathrm{U} / \mathrm{mL}$, but other autoantibodies including antibasement membrane antibody were negative. Serum cryoglobulin was negative. Hematuria of 20 red blood cells per high-power field was confirmed by examination of urinary sediment, and proteinuria was positive.

Chest X-ray showed a mass-like shadow in the right hilum and nodules (Fig. 1). Chest computed tomography (CT) showed a mass lesion of $55 \mathrm{~mm}$ in diameter in the apical segment of the right lower lobe (Fig. 1). Multiple nodules and ground-glass opacities were found in both lung fields. Bronchoscopy showed a skip-lesion of mucosal erosion with a white necrotic substance that extended from the trachea into the right main bronchus (Fig. 2). A biopsy specimen of the erosion showed ulcerated bronchial mucosa with necrotic tissue and atypical regeneration of squamous epithelium (Fig. 3). Bronchoalveolar lavage fluid was bloody, and hemosiderin-laden macrophages were seen. A TBLB specimen from the right lower lobe showed hemosiderin-laden macrophages, small abscess-like lesions, and fibrin exudates in the alveolar tissue (Fig. 4a). The presence of partial destruction of the alveolar wall was revealed in the abscesslike lesion by specific stains and immunohistochemical staining (Fig. 4b-d). Neutrophilic capillaritis was confirmed.

On the basis of the clinical presentation, positive results of PR-3 ANCA, and bronchoscopic findings including histologic evidence of capillaritis, we diagnosed her as having pulmonary capillaritis and DAH due to WG on the 4th hospital day. Methylprednisolone at a daily dose of $1 \mathrm{~g}$ was administered from the 4th to the 6th hospital day, followed by $60 \mathrm{mg}$ of prednisolone daily. Cyclophosphamide at a daily 


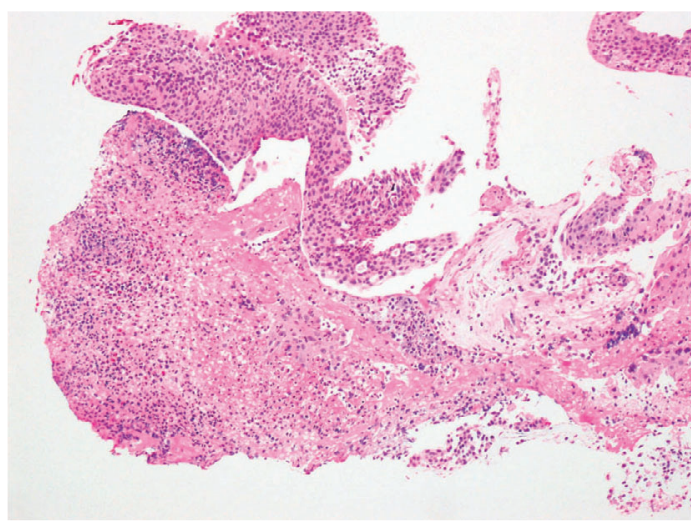

a

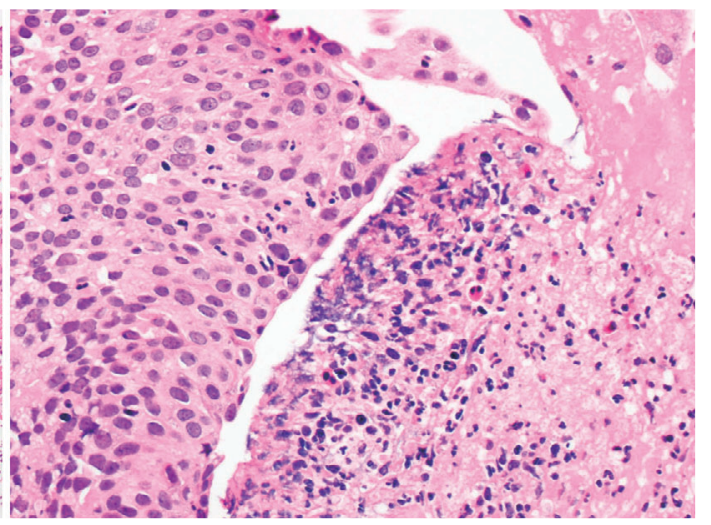

$\mathrm{b}$

Figure 3. Histologic findings obtained via transbronchial biopsy of the tracheal erosion. Necrotic tissue and mild atypical squamous cells were seen (Hematoxylin and Eosin staining, a. $\times 200$, b. $\times \mathbf{8 0 0})$.
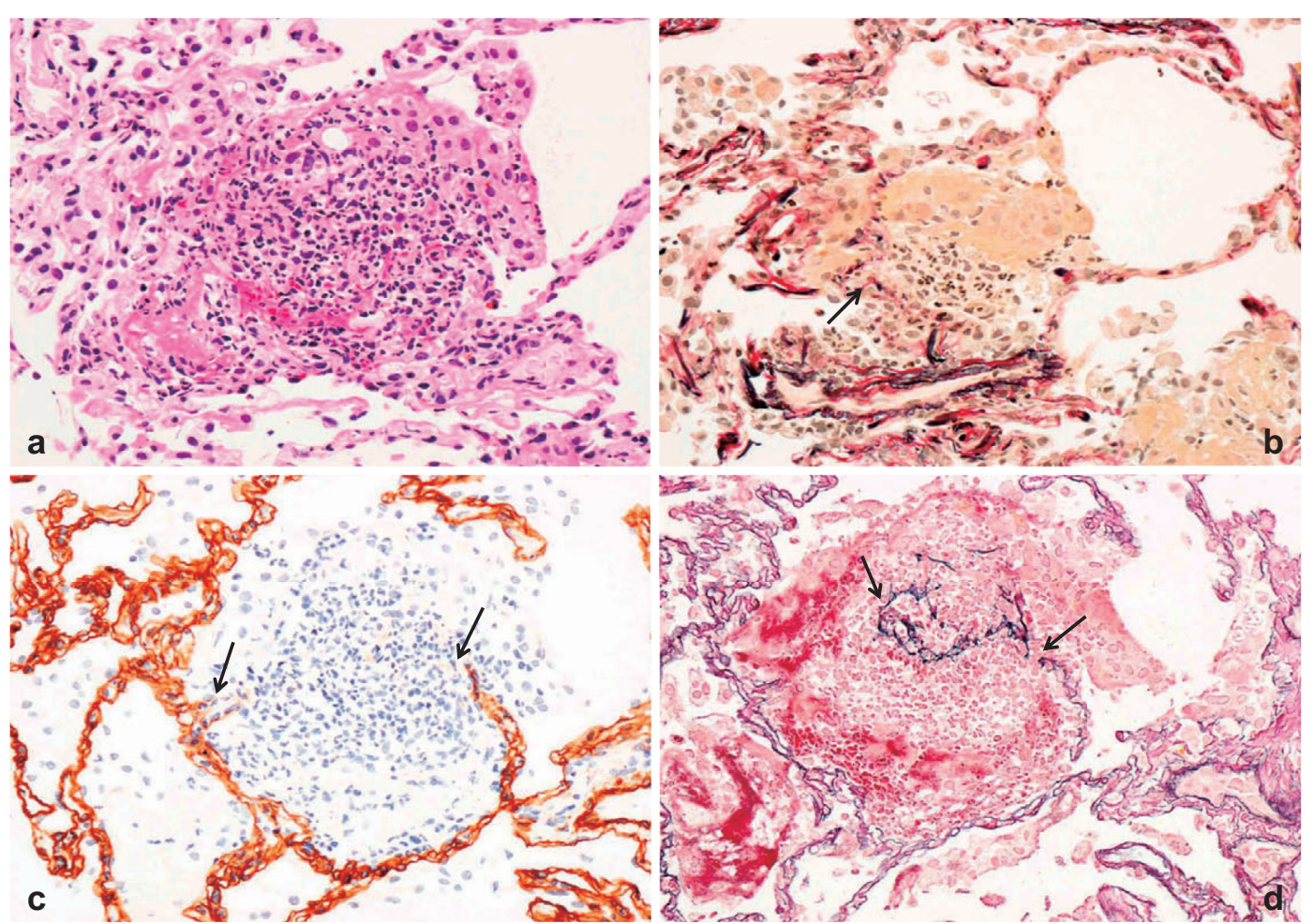

Figure 4. Histologic findings obtained via transbronchial lung biopsy. A transbronchial lung biopsy specimen from the right lower lobe showed exudation of neutrophils and fibrin in the alveolar tissue (a, Hematoxylin and Eosin staining, $\times 300$ ). This exudation originated from the alveolar wall (b, arrow, Elastica van Gieson staining, $\times 300$ ). Type IV collagen and reticulin fibers showed disruption in this area (c, immunostaining using antibody for type IV collagen, $\times 300, d$, silver impregnation, $\times 300$ ). These findings suggest that neutrophils destroyed the alveolar wall and resulted in exudation of cells (and blood) and fluid including fibrinogen into the alveolar spaces. Histologically, this was diagnosed as neutrophilic capillaritis. Arrows indicate the disrupted portions of the alveolar wall (bd).

dose of $100 \mathrm{mg}$ was added from the 5th hospital day. Scleritis of her right eye was resolved; however, hemoptysis continued. From the 8th to 10th hospital day, methylprednisolone $1 \mathrm{~g}$ daily was readministered, but the hemoptysis was not resolved. Her respiratory condition and anemia worsened with $\mathrm{aaO}_{2}$ of $65.2 \mathrm{mmHg}$ under $5 \mathrm{~L} / \mathrm{min}$ oxygen and hemoglobin of $6.5 \mathrm{~g} / \mathrm{dL}$, thus we performed plasmapheresis from the 10th to 12th hospital day, and hemoptysis stopped on the 11th hospital day. While continuing administration of sulfamethoxazole-trimethoprim $1 \mathrm{~g}$ daily, the prednisolone 


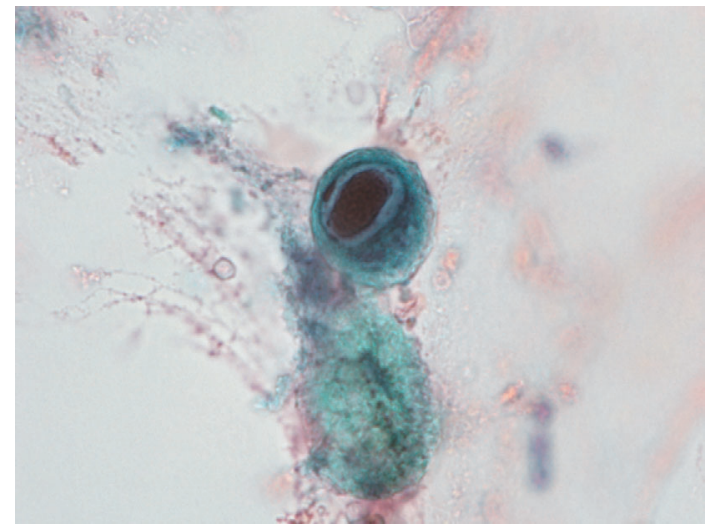

Figure 5. Cytology of bronchial washing fluid. Bronchial washing fluid showed cells containing reddish brown-colored large inclusion bodies in their nuclei (Papanicolaou stain, $\times 1,200)$.

dose was gradually tapered by $10 \mathrm{mg}$ every 2 weeks, and cyclophosphamide $100 \mathrm{mg}$ daily was continued. From the 26th hospital day, the patient developed abdominal discomfort and nausea. On the 30th hospital day, her respiratory condition rapidly deteriorated with fever, and ground-glass opacities re-appeared in both lung fields. The patient was intubated and mechanically ventilated. Bronchial aspirate did not yield significant microorganisms, but bronchial washing fluid showed cells containing reddish brown-colored large inclusion bodies in their nuclei (Fig. 5), highly suggestive of cytomegalovirus (CMV) infection. CMV infection was also suggested by positive results of CMV pp65 antigenemia assay with 58 positive cells per $5.0 \times 10^{4}$ polymorphonuclear leukocytes and peripheral blood lymphocytopenia of 100/ $\mathrm{mm}^{3}$. Beta-D glucan was not elevated on the 36th hospital day. Serum PR-3 ANCA levels were decreased to within the normal range. Administration of ganciclovir $250 \mathrm{mg}$ daily was begun, but the patient died on the 41 st hospital day.

\section{Discussion}

We report a case of WG in which DAH developed due to capillaritis. Bronchoscopy showed endobronchial involvement in which specific histologic findings of WG were not obtained. TBLB did not show necrotizing granulomatous inflammation, but capillaritis with alveolar hemorrhage was detected.

Respiratory involvement of WG occurs in the upper airways and lungs. Endobronchial lesions occur in about $50 \%$ of WG cases $(1,3)$. Daum et al performed bronchoscopy in 51 patients with biopsy-proven WG, and 30 (59\%) patients had endobronchial abnormalities (3). Their findings included subglottic stenosis in $5(17 \%)$, ulcerating tracheobronchitis with or without inflammatory pseudotumors in $18(60 \%)$, tracheal or bronchial stenosis without inflammation in 4 (13\%), and hemorrhage without identifiable source in 2 (4\%) patients. However, among 21 specimens from 17 patients with WG, only 3 specimens from 3 different patients were diagnostic of WG. All but 3 specimens indicated inflammatory activity (3). Allen and Moen performed transbronchial biopsy in 16 patients, but specific pathologic findings were not detected (4). In the present patient, we performed endobronchial biopsy of the erosion of the bronchial mucosa; however, we could only detect ulcerative tracheobronchitis with necrotic tissue and atypical regeneration of squamous epithelium, which were not diagnostic for WG. It may be difficult to obtain a biopsy specimen of an endobronchial lesion that can yield specific pathological findings.

Histologic findings of the lung in WG vary; however, the changes due to WG are approached by dividing them into three histologic categories: necrotizing granulomatous inflammation with prominent parenchymal necrosis and necrotizing vasculitis, organizing pneumonia-like variant, and alveolar hemorrhage and capillaritis (5). To obtain pulmonary samples, surgical lung biopsy (open lung biopsy and thoracoscopy) and TBLB are generally performed. Surgical lung biopsy is regarded as a standard procedure for diagnosing WG. Although TBLB is a less morbid procedure than surgical lung biopsy, reports in which TBLB was found to be a useful diagnostic procedure for WG were limited to sporadic case reports $(6,7)$ and a small-sized case series (8). Pinching et al proved the diagnosis of WG in 2 of 7 patients via TBLB (6). Unfortunately, we could not detect necrotizing granulomatous inflammation or necrotizing vasculitis in the present patient, but we were able to detect DAH and capillaritis via TBLB.

Pulmonary capillaritis has been reported to be found in 17 to $43 \%$ of patients with WG who underwent open lung biopsy $(9,10)$. Although there have been reports of WG presenting with DAH due to capillaritis (11-13), there have been no reports of capillaritis detected via TBLB, and the frequency at which TBLB detects capillaritis remains unknown. The reasons for the absence of such reports may be due to the difficulty in both detecting capillaritis in the small specimens obtained via TBLB and in performing TBLB because patients are too ill. Although such problems may exist, TBLB is a less morbid procedure than surgical lung biopsy, and we could detect capillaritis in a TBLB specimen.

A variety of diseases are associated with pulmonary capillaritis, including WG, microscopic polyangiitis, acute lupus pneumonitis, drug reactions, and others (5). Therefore, etiology should be differentiated when histologic findings of capillaritis are detected. In the present patient, scleritis, hearing difficulty, an upper airway lesion detected via bronchoscopy, DAH due to capillaritis, hematuria and proteinuria, and positivity of PR-3 ANCA lead to the diagnosis of WG (14).

The prevailing standard regimen for severe disease that includes DAH due to capillaritis consists of the combined use of oral cyclophosphamide ( $2 \mathrm{mg} / \mathrm{kg} /$ day $)$ and prednisolone $(1 \mathrm{mg} / \mathrm{kg} / \mathrm{day})$, and complete remission can be achieved in over $90 \%$ of patients. In resistant cases, plasmapheresis is 
added. Several studies have suggested the effectiveness of plasmapheresis in the management of severe ANCAassociated vasculitis manifested by DAH. Frascà et al examined the combined effects of plasmapheresis and drugs (steroids and cyclophosphamide) versus the drugs alone in 26 patients with ANCA-associated crescentic glomerulonephritis (15), and they found that the combined therapy resulted in a significantly improved prognosis. In the present case, hemoptysis resolved 1 day after plasmapheresis, indicating good efficacy.

The fact that up to $46 \%$ of patients treated with a combination regimen of glucocorticoids and cyclophosphamide were reported to suffer serious infectious complications is problematic (2). CMV-related disease is a relatively frequent complication of immunosuppressive treatment for systemic vasculitis such as WG (16-18). In the present patient, gastrointestinal symptoms, positivity of CMV antigenemia assay on the 36th hospital day, detection of cells containing inclusion bodies in their nuclei in bronchial washings, and diffuse ground-glass opacities on chest CT were found, all of which suggest CMV infection.

In conclusion, we could detect capillaritis in a TBLB specimen obtained from a patient with WG. TBLB is a less morbid procedure than that of surgical lung biopsy; therefore, TBLB should be considered when attempting to obtain tissue from a patient with pulmonary disease and suspected WG.

The authors state that they have no Conflict of Interest (COI).

\section{References}

1. Cordier JF, Valeyre D, Guillevin L, et al. Pulmonary Wegener's granulomatosis. A clinical and imaging study of 77 cases. Chest 97: 906-912, 1990.

2. Hoffman GS, Kerr GS, Leavitt RY, et al. Wegener granulomatosis: an analysis of 158 patients. Ann Intern Med 116: 488-498, 1992.

3. Daum TE, Specks U, Colby TV, et al. Tracheobronchial involvement in Wegener's granulomatosis. Am J Respir Crit Care Med 151: 522-526, 1995.

4. Allen AR, Moen CE. Wegener's granulomatosis. Case report and evaluation of the diagnostic techniques used in disease of the chest. J Thorac Cardiovasc Surg 49: 388-397, 1965.

5. Katzenstein AL. Katzenstein and Askin's Surgical Pathology of Non-neoplastic Lung Disease. Fourth ed. Saunders, Philadelphia, 2006: 217-236.

6. Pinching AJ, Lockwood CM, Pussell BA, et al. Wegener's granulomatosis: observations on 18 patients with severe renal disease. Q J Med 52: 435-460, 1983.

7. Ackerman Z, Orbach H, Burstein M, et al. Transbronchial biopsies in Wegener's granulomatosis. Ann Intern Med 105: 801-802, 1986.

8. Givens CD Jr, Newman JH, McCurley TL. Diagnosis of Wegener's granulomatosis by transbronchial biopsy. Chest 88: 794-796, 1985.

9. Mark EJ, Matsubara O, Tan-Liu N, et al. The pulmonary biopsy in the early diagnosis of Wegener's (pathergic) granulomatosis: a study based on 35 open lung biopsies. Hum Pathol 19: 10651071, 1988.

10. Travis WD, Hoffman GS, Leavitt RY, et al. Surgical pathology of the lung in Wegener's granulomatosis. Review of 87 open lung biopsies from 67 patients. Am J Surg Pathol 15: 315-333, 1991.

11. Mark EJ, Ramirez JF. Pulmonary capillaritis and hemorrhage in patients with systemic vasculitis. Arch Pathol Lab Med 109: 413418, 1985.

12. Yoshimura N, Matsubara O, Tamura A, et al. Wegener's granulomatosis. Associated with diffuse pulmonary hemorrhage. Acta Pathol Jpn 42: 657-661, 1992.

13. Stokes TC, McCann BG, Rees RT, et al. Acute fulminating intrapulmonary haemorrhage in Wegener's granulomatosis. Thorax 37: 315-316, 1982.

14. Leavitt RY, Fauci AS, Bloch DA, et al. The American College of Rheumatology 1990 criteria for classification of Wegener's granulomatosis. Arthritis Rheum 33: 1101-1107, 1990.

15. Frascà GM, Soverini ML, Falaschini $A$, et al. Plasma exchange treatment improves prognosis of antineutrophil cytoplasmic antibody-associated crescentic glomerulonephritis: a case-control study in 26 patients from a single center. Ther Apher Dial 7: 540546, 2003.

16. Sackier JM, Kelly SB, Clarke D, et al. Small bowel haemorrhage due to cytomegalovirus vasculitis. Gut 32: 1419-1420, 1991.

17. Woywodt A, Choi M, Schneider W, et al. Cytomegalovirus colitis during mycophenolate mofetil therapy for Wegener's granulomatosis. Am J Nephrol 20: 468-472, 2000.

18. Yamazaki $M$, Takei $T$, Otsubo $S$, et al. Wegener's granulomatosis complicated by intestinal ulcer due to cytomegalovirus infection and by thrombotic thrombocytopenic purpura. Intern Med 46: 1435-1440, 2007.

(C) 2012 The Japanese Society of Internal Medicine http://www.naika.or.jp/imindex.html 